\section{TAB NEWS}

\section{Neue Themen in der 18. Legislaturperiode}

Nachdem im Frühjahr 2014 die Vorsitzende des Ausschusses für Bildung, Forschung und Technikfolgenabschätzung (ABFTA), Patricia Lips, eine Themenfindungsrunde für neue TA-Projekte bei allen Ausschüssen und Fraktionen des Deutschen Bundestages gestartet hatte, gingen bis Anfang Mai gut 40 Untersuchungsanträge beim ABFTA ein. Alle Themenvorschläge wurden vom $\mathrm{TAB}$ gemeinsam mit den neuen Kooperationspartnern eingehend kommentiert, um der „Berichterstattergruppe TA“ Vorschläge für eine mögliche Bearbeitung zu unterbreiten. Nach einer intensiven gemeinsamen Diskussion im Rahmen des interfraktionellen TA-Berichterstattergesprächs Anfang Juni 2014 wurde von den Abgeordneten eine Themenstaffel von elf Projekten ausgewählt (zuzüglich dreier Themen, die in Form von Gesprächsforen oder Workshops behandelt werden). Grundsätzlich befürwortet wurden sechs weitere Themen, die nach einer entsprechenden Beschlussfassung im Lauf der Jahre 2015/2016 vom TAB bearbeitet werden könnten.

In seiner letzten Sitzung vor der Sommerpause hat der ABFTA die neuen Projekte beschlossen. Es handelt sich um folgende Themen (Kurztitel), die im kommenden TAB-Brief Nr. 44 (September 2014) kurz vorgestellt werden:

- Online-Bürgerbeteiligung an der Parlamentsarbeit

- Neue elektronische Medien und Suchtverhalten

- Digitale Medien in der Bildung

- Künstliche Intelligenz (Mensch-MaschineEntgrenzungen)

- Pharmaka in Gewässern

- Digitales Arbeiten

- Data-Mining

- Umgang mit Nichtwissen

- Interessenausgleich bei Infrastrukturprojekten

- Bilanz der Sommerzeit

- Additive Fertigungsverfahren/3D-Druck

Im Kontext dieser Projekte wird ab sofort und kontinuierlich bis in den Herbst hinein auf der TAB-Homepage (http://www.tab-beim-bundes- tag.de) unter der Rubrik „Gutachter gesucht" sowie „Aktuelles“ über die Vergabe von Gutachten informiert.

\section{$\ll »$}

Öffentliche Ausschusssitzung zum TABBericht „Climate Engineering“ am 24. September 2014 im Deutschen Bundestag

Der Klimawandel wird vielfach als das zentrale Umweltproblem unserer Zeit angesehen. Zur Beherrschung der Klimaveränderungen und ihrer vielfältigen Folgen verfolgt die Weltgemeinschaft seit Längerem verschiedene Strategien zur Reduktion anthropogener Treibhausgasemissionen (Mitigation) sowie Anpassungsmaßnahmen (Adaption) an eingetretene oder erwartete Klimaänderungen. Ungeachtet der vielfältigen Klimaschutzbemühungen ist es bisher nicht gelungen, den weiteren Anstieg der Treibhausgaskonzentrationen in der Atmosphäre aufzuhalten. Um der damit verbundenen Erderwärmung entgegenzuwirken, gibt es prinzipiell noch andere Handlungsoptionen. Dazu gehören gezielte technische Interventionen in das Klimasystem - das sog. Climate Engineering. Hierbei soll durch Technikeinsatz in großen und größten Dimensionen entweder das bereits emittierte $\mathrm{CO}_{2}$ wieder aus der Atmosphäre entfernt oder direkt in den Strahlungshaushalt der Erde eingegriffen werden.

Ob bzw. unter welchen Bedingungen Climate Engineering einen Beitrag zur Verhinderung eines unter Umständen folgenschweren Klimawandels liefern könnte oder gar sollte, diese Frage steht im Mittelpunkt des Endberichts zum TA-Projekt „Climate Engineering“ (s. „Neue Veröffentlichungen“ TAB-Arbeitsbericht Nr. 159). Der Bericht gibt einen umfassenden Überblick über den Stand des Wissens bezüglich naturwissenschaftlichtechnologischer Aspekte der vorgeschlagenen Ansätze des Climate Engineering. Darüber hinaus werden die rechtlichen Rahmenbedingungen und Regulierungserfordernisse in nationaler und internationaler Perspektive analysiert. Schließlich wird der Blick auf ethische, politische und sozioökonomische Bewertungskriterien gerichtet, die angesichts der zu erwartenden weitreichenden Konsequenzen einer großskaligen Anwendung von Climate Engineering von besonderer Relevanz 
für Entscheidungen über weitere Forschungs- und Entwicklungsaktivitäten sind.

Angesichts der Bedeutung des Themas für die Klimaschutzpolitik hat der ABFTA beschlossen, die Präsentation der Ergebnisse des Abschlussberichts zum TA-Projekt im Rahmen einer öffentlichen Ausschusssitzung am 24. September 2014 durchzuführen. Zur Diskussion der vom TAB gezogenen Schlussfolgerungen sind neben den Ausschussmitgliedern interessierte Bundestagsabgeordnete aus anderen Ausschüssen, Experten aus der Wissenschaft und den Ministerien sowie die interessierte Öffentlichkeit eingeladen. Ziel der Veranstaltung mit dem Titel „Climate Engineering - sinnvolles Instrument oder Sackgasse in der Klimapolitik?" ist es, eine dringend gebotene gesellschaftspolitische Debatte über die Sinnhaftigkeit von Climate Engineering anzuregen und zugleich weitere notwendige (politische) Schritte im Umgang mit Climate Engineering zu identifizieren.

Die öffentliche Ausschusssitzung findet am 24. September 2014 von 15 bis 17.30 Uhr im Sitzungssaal E 300 des Paul-Löbe-Hauses statt. Das Veranstaltungsprogramm sowie das Formular für die Online-Anmeldung werden in den kommenden Wochen auf den Internetseiten des TAB (http://www.tab-beim-bundestag.de) zur Verfügung gestellt.

\section{$\ll 》$}

\section{Weitere TAB-Berichte im Bundestag}

Am 2. Juli 2014 nahm der ABFTA den TAB-Arbeitsbericht Nr. 159 „Climate Engineering“ ab. Der TAB-Arbeitsbericht Nr. 156 „Postdienste und moderne Informations- und Kommunikationstechnologien" ist als Bundestagsdrucksache Nr. 18/582 erschienen und am 22. Mai 2014 im Plenum behandelt worden. Die zu Protokoll gegebenen Reden sind im Plenarprotokoll 18/36, S. 3170-3175 abgelegt. Folgende TAB-Arbeitsberichte wurden vom ABFTA mit Kenntnisnahme abschließend beraten:

- „Konzepte der Elektromobilität und deren Bedeutung für Wirtschaft, Gesellschaft und Umwelt“ (Bericht Nr. 153) und „Zukunft der Automobilindustrie" (Bericht Nr. 152) am 2. Juli 2014
- „Gesetzliche Regelungen für den Zugang zur Informationsgesellschaft" (Bericht Nr. 149) am 7. Mai 2014

- „Wettbewerbsfähigkeit der europäischen Wirtschaft im Hinblick auf die EU-Beihilfepolitik - am Beispiel der Nanoelektronik“" (Bericht Nr. 139) am 2. April 2014

\section{$\ll 》$}

\section{TAB-Fokus: Ergebnisse kurz und bündig}

Eine kurze, anschauliche und dennoch fundierte Zusammenfassung wichtiger Ergebnisse von TA-Analysen stand schon lange auf unserer Vorhabensliste. Die Neuaufstellung des TAB in der aktuellen Vertrags- und Wahlperiode gab nun den endgültigen Anstoß, das neue Publikationsformat TAB-Fokus zu entwickeln. Diese vierseitigen Kurzinformationen sollen zukünftig zu allen Abschlussberichten und fallweise z. B. anlässlich öffentlicher Veranstaltungen oder sonstiger besonderer Ereignisse erscheinen.

Die ersten Ausgaben sind seit Kurzem auf der Webseite verfügbar: TAB-Fokus Nr. 1 zum Arbeitsbericht Nr. 154 „Fernerkundung: Anwendungspotenziale in Afrika“, TAB-Fokus Nr. 2 zum Arbeitsbericht Nr. 156 ,Postdienste und moderne Informations- und Kommunikationstechnologien“" und TAB-Fokus Nr. 3 zum Arbeitsbericht Nr. 159 „Climate Engineering“. Wir freuen uns über das Ergebnis, das auch von den neuen Berichterstattern für TA im Deutschen Bundestag begrüßt wurde. Wir hoffen, dass ein ähnlich positiver Eindruck bei den zukünftigen Lesern und Nutzern entsteht, und sind selbstverständlich offen für Anregungen zur Verbesserung und Weiterentwicklung.

Gedruckte Exemplare werden in Zukunft den TAB-Arbeitsberichten beigelegt, elektronisch werden TAB-Fokus-Ausgaben auf den jeweiligen Projektseiten angeboten.

\section{$\ll 》$}

\section{Das TAB verstärkt die internationale Sichtbarkeit ausgewählter Studien}

Obwohl das Büro für Technikfolgen-Abschätzung beim Deutschen Bundestag (TAB) seinen ersten Adressaten (und Auftraggeber) im Deutschen Bundestag hat, wurde von Anfang an auch 
auf die internationale Sichtbarkeit der Ergebnisse der TAB-Studien Wert gelegt. Ganz explizit ist eine Teilnahme und Zusammenarbeit des TAB im „European Parliamentary Technology Assessment Network" (EPTA-Netzwerk) mit dem Ziel einer Stärkung der internationalen parlamentarischen TA auch über Europa hinaus in den Grundsätzen der TA festgeschrieben worden.

Denn nicht nur die wissenschaftlichen Debatten finden im internationalen Rahmen statt, sondern auch forschungs- und technologiepolitische Entscheidungen sind immer mehr in internationalen Institutionen verankert, am augenscheinlichsten bei der Europäischen Union, aber auch in vielen weiteren internationalen politischen Institutionen. Die Verfügbarkeit und Rezipierbarkeit der TAB-Studien in diesen Gremien wird deshalb immer wichtiger.

Über die früh etablierte Webseite des TAB konnten schon immer die ausführlichen Zusammenfassungen der TAB-Berichte auch in englischer Sprache abgerufen werden. Seit 2009 hat das $\mathrm{TAB}$ darüber hinaus mit Zustimmung der Berichterstattergruppe für TA eine eigene englischsprachige Buchreihe etabliert, in der ausgewählte TABBerichte, die ein besonderes internationales Interesse erwarten lassen, aufgenommen werden. Diese Buchreihe umfasst zurzeit sechs Titel und ist über die Website des TAB direkt abrufbar (http://www. tab-beim-bundestag.de/en/publications/books/).

Mit dem Erscheinen des 6. Bandes „Electronic petitioning and modernization of petitioning systems in Europe" - ein Thema, das derzeit bei verschiedenen europäischen Parlamenten virulent ist - wurde eine weitere Neuerung eingeführt: Die Titel der englischen TAB-Buchreihe stehen nun auch weltweit als gedrucktes Buch zu sehr moderaten Preisen über den Online- oder stationären Buchhandel zur Verfügung. Die aktuelle Liste der englischen TAB-Buchreihe:

- Riehm, Ulrich; Böhle, Knud; Lindner, Ralf: Electronic petitioning and modernization of petitioning systems in Europe. Norderstedt: 2014, Paperback, 296 Seiten, ISBN 978-37357-9033-0, 14,98 Euro (22,90 US\$)

- Sauter, Arnold; Gerlinger, Katrin: The pharmacologically improved human. Norderstedt: 2013, Paperback, 296 Seiten, ISBN 978-37322-9681-1, 14,99 Euro (22,50 US\$)
- Petermann, Thomas; Bradke, Harald; Lüllmann, Arne; Poetzsch, Maik; Riehm, Ulrich: What Happens During a Blackout. Norderstedt: 2011, Paperback, 252 Seiten, ISBN 978-3-7322-9329-2, 12,75 Euro (19,50 US\$)

- Gerlinger, Katrin; Petermann, Thomas; Sauter, Arnold: Gene Doping. Norderstedt: 2009, Paperback, 156 Seiten, ISBN 978-3-73228785-7, 7,92 Euro (12,50 US\$)

- Grünwald, Reinhard: Greenhouse Gas - Bury it into Oblivion. Norderstedt: 2009, Paperback, 132 Seiten, ISBN 978-3-7322-8815-1, 6,70 Euro (10,90 US\$)

- Coenen, Christopher; Riehm, Ulrich: Development Through Electronic Networks. Norderstedt: 2009, Paperback, 268 Seiten, ISBN 978-3-7322-6212-0, 13,58 Euro (19,90 US\$)

(Preisangaben sind nur als Orientierung zu verstehen, da in Ländern ohne Buchpreisbindung die Verkaufspreise variieren können.)

\section{$\ll 》$}

\section{Neue Veröffentlichungen}

TAB-Arbeitsbericht Nr. 159 „Climate Engineering “ (März 2014; Verfasser: Claudio Caviezel, Christoph Revermann)

Zur Eindämmung des Klimawandels und seiner vielfältigen Auswirkungen verfolgt die Weltgemeinschaft seit Längerem verschiedene Strategien zur Reduktion anthropogener Treibhausgasemissionen sowie Anpassungsmaßnahmen an eingetretene oder erwartete Klimafolgen. Ungeachtet der vielfältigen Klimaschutzbemühungen ist es bisher jedoch nicht gelungen, den weiteren Anstieg der Treibhausgaskonzentrationen in der Atmosphäre aufzuhalten. Vor diesem Hintergrund gewinnen die Diskussionen um mögliche gezielte technische Interventionen in das Klimasystem zur Beherrschung des Klimawandels - das sog. Climate Engineering - immer weiter an Fahrt. Der mögliche Nutzen von Climate Engineering wird aber - selbst unter denjenigen, die es vorschlagen -, sehr kontrovers diskutiert, denn klar ist, dass technische Maßnahmen, die von ihrer Anlage her eine weiträumige bis globale Manipulation der natürlichen Erdsystemprozesse beabsichtigen, zugleich mit mannigfaltigen Auswirkungen für Mensch und Umwelt verbunden wären. 
Ob bzw. unter welchen Bedingungen Climate Engineering einen Beitrag zur Verhinderung eines unter Umständen folgenschweren Klimawandels liefern könnte oder gar sollte, diese Frage steht im Mittelpunkt des TAB-Berichts. Er gibt einen umfassenden Überblick über den Stand des Wissens bezüglich naturwissenschaftlich-technologischer Aspekte des Climate Engineering. Darüber hinaus werden die rechtlichen Rahmenbedingungen und Regulierungserfordernisse in nationaler und internationaler Perspektive analysiert. Schließlich wird der Blick auf ethische, politische und sozioökonomische Bewertungskriterien gerichtet, die angesichts der zu erwartenden weitreichenden Konsequenzen einer großskaligen Anwendung von Climate Engineering von besonderer Relevanz für Entscheidungen über weitere Forschungs- und Entwicklungsaktivitäten sind.

Weil die Optionen des Climate Engineering den klimapolitischen Handlungsspielraum prinzipiell und in vielfacher Hinsicht grundlegend erweitern, gibt es keine einfachen Antworten auf die zuvor genannte Frage. Vielmehr wird die dringende Notwendigkeit für eine breite gesellschaftspolitische Debatte darüber, ob bzw. welche Ansätze des Climate Engineering weiter erforscht (und gegebenenfalls zur Anwendungsreife gebracht) und welche Risiken dafür eingegangen werden sollen, sehr deutlich. Der TAB-Bericht soll dafür relevante Grundlagen verdeutlichen.

TAB-Arbeitsbericht Nr. 158 „Herausforderungen einer nachhaltigen Wasserwirtschaft " (Mai 2013; Verfasser: Thomas Hillenbrand, Harald Hiessl, Stefan Klug, Benedikt Freiherr von Lüninck, Jutta Niederste-Hollenberg, Christian Sartorius, Rainer Walz)

Das Zusammenspiel aus demografischen, klimatischen und wirtschaftsstrukturellen Wandelprozessen stellt die Wasserwirtschaft vieler Weltregionen gegenwärtig vor große Herausforderungen. Während im Kontext des Klimawandels gravierende Veränderungen regionaler Wasserkreisläufe erwartet werden, steigt zeitgleich der Nahrungsmittelund Energiebedarf einer global wachsenden Bevölkerung. Vielerorts verschärft sich dadurch das Spannungsfeld konfliktärer Nutzungsinteressen an den Wasserressourcen. Gleichzeitig ergeben sich zusätzliche Herausforderungen hinsichtlich der Verunreinigungen der Gewässer mit Nährstoffen und Schadstoffen, wie z. B. Schwermetalle, Pestizide und andere organische Mikroschadstoffe.

Mit den Rahmenbedingungen ändern sich in den kommenden Jahrzehnten auch die Anforderungen an die Wasserinfrastrukturen teilweise drastisch. Die überwiegend leitungsgebundenen und damit sehr langlebigen und inflexiblen, gleichzeitig aber auch kapitalintensiven Infrastrukturen zur Wasserver- und Abwasserentsorgung werden teilweise an ihre Leistungs- bzw. Wirtschaftlichkeitsgrenzen kommen - sie müssen deshalb rechtzeitig an die zukünftigen Herausforderungen angepasst und flexiblere Lösungskonzepte entwickelt und umgesetzt werden.

Vor diesem Hintergrund wird im Rahmen des TAB-Berichts die weltweite Innovationsdynamik im Bereich Wasser untersucht, das Innovationssystem im Bereich der Wassertechnologien analysiert, und es werden wichtige Herausforderungen und Trends für Industrie-, Schwellen- und Entwicklungsländer aufgezeigt.

International zeichnen sich deutsche Hersteller wasserwirtschaftlich relevanter Technologiegüter durch eine hochsignifikante Spezialisierung in allen Technikbereichen und den weltweit höchsten Außenhandelsanteilen aus. Diese Leistungsfähigkeit war im vergangenen Jahrzehnt und ist bis heute eine der Stützen der deutschen Außenhandelserfolge. Marktprognosen gehen aufgrund des großen globalen Bedarfs von einem Markt mit hohen Wachstumsraten aus. Aufgrund der jüngsten Entwicklung bei der technologischen Leistungsfähigkeit der deutschen Hersteller - deutlicher Rückgang der Anteile an Patentanmeldungen und Publikationen - ist allerdings zu befürchten, dass mittel- bis langfristig die internationale Wettbewerbsfähigkeit der deutschen Hersteller zurückgehen und auch der Außenhandelsanteil abnehmen wird. In Deutschland sind deshalb verstärkte Anstrengungen zur Förderung des Innovationssystems im Bereich der Wassertechnologien notwendig. Dies betrifft die Forschungsförderung, die gezielte Förderung des Übergangs von Forschungsergebnissen in die Praxis sowie die Stärkung und dauerhafte Unterstützung der internationalen Wettbewerbsfähigkeit.

\section{$\langle\gg$}

